Research Paper

\title{
An Application of NGS for WDR36 Gene in Taiwanese Patients with Juvenile-Onset Open-Angle Glaucoma
}

\author{
Hsuan-An Su1, 2, Shuan-Yow Li 3,4, Jiann-Jou Yang ${ }^{3,4 \bowtie}$, Yung-Chang Yen ${ }^{5,6 \bowtie}$ \\ 1. Department of Medical Education, Chung Shan Medical University Hospital, Taichung, Taiwan; \\ 2. School of Medicine, Chung Shan Medical University, Taichung, Taiwan; \\ 3. Department of BioMedical Sciences, Chung Shan Medical University, Taichung, Taiwan; \\ 4. Department of Medical Research, Chung Shan Medical University Hospital, Taichung, Taiwan; \\ 5. Department of Ophthalmology, Chi-Mei Medical Center, Liou-Ying, Tainan, Taiwan; \\ 6. Department of Nursing, Min Hwei Junior College of Health Care Management, Tainan, Taiwan
}

$\triangle$ Corresponding authors: Dr. J-J Yang, Department of BioMedical Sciences, Chung Shan Medical University, Taichung, Taiwan Tel:886-4-24730022, ext. 11804; Fax: 886-4-24757412; E-mail: jiannjou@csmu.edu.tw; Dr. Y-C Yen, Department of Ophthalmology, Chi-Mei Medical Center, Liou-Ying, Tainan, Taiwan Tel:886-6-6226999 E-mail: dy5101@yahoo.com.tw

(c) Ivyspring International Publisher. This is an open access article distributed under the terms of the Creative Commons Attribution (CC BY-NC) license (https://creativecommons.org/licenses/by-nc/4.0/). See http://ivyspring.com/terms for full terms and conditions.

Received: 2017.04.25; Accepted: 2017.08.07; Published: 2017.09.20

\begin{abstract}
Primary open-angle glaucoma (POAG) is one of the most important disease in ophthalmology with high prevalence and risk of irreversible blindness. If diagnosed before the age of 35 , it is usually categorized as juvenile open-angle glaucoma (JOAG). The WDR36 gene is reckoned as one of the major causative genes of POAG, and had been studied to be related to the pathogenesis of POAG in the literature. We have selected 61 JOAG patients and 61 JOAG-free individuals, and by next-generation sequencing method, the WDR36 gene of the subjects were analyzed. We identified 26 variations exclusively in JOAG group. Among these 26 variations, there were 3 noteworthy variations. First, a novel variation c. $460-650 \mathrm{~A}>\mathrm{G}$ was found in our study which might cause premature termination of splicing of the conserved domain in WDR36; second, c.1494+1111G>T (rs13178997) had significantly different frequency in our JOAG patients compared to the reference frequency on NCBl; third, a variation c.710+30C >T ( $\mathrm{rs} 10038177$ ) was found in our study, which had already been reported to be related to high-pressure glaucoma. We offer the profile of WDR36 in JOAG in Taiwan population, and we suggest that WDR36 gene is involved in the pathogenesis of JOAG as a subordinate modifier gene.
\end{abstract}

Key words: WDR36, polymorphism, glaucoma, JOAG, mutation.

\section{Introduction}

Primary open-angle glaucoma (POAG) is a major type of glaucoma worldwide, a progressive optic neuropathy typically featuring increased intraocular pressure (IOP) and degeneration of retinal ganglion cells and fibers, with high risk of irreversible blindness [1, 2]. In patients younger than 35 years old, open-angle glaucoma is often diagnosed as juvenile open-angle glaucoma (JOAG) [3]. The etiology of JOAG remains unknown, but it is believed that the pathogenesis of glaucoma is multifactorial in genetic, environmental, cardiovascular, intraocular pressure-related and aging aspects, with the genetic etiology of glaucoma mostly studied $[4,5]$.

POAG is a critical ophthalmic issue worldwide, with over a half of all POAG patients in Asia. By 2020, it was estimated that over 73 million people would be affected by POAG [6]. In a cross-sectional survey of Chinese population over 40 years old in Singapore, $3.2 \%$ prevalence of POAG was observed and POAG was reported to be the leading cause of blindness [7]. In another study, glaucoma is also shown to be the leading cause of low vision and blindness in Taiwan [8]. Thus, POAG is increasingly concerned as a critical issue in ophthalmology due to its high prevalence and severe optic sequelae.

POAG was heterogeneously associated with at least 20 genetic loci, and among these loci, 11 loci were denominated as GLC1A to GLC1K, among which GLC1A, GLC1E, GLC1G were further identified as myocilin (MYOC), optineurin (OPTN), WD repeat 
domain 36 (WDR36) genes, respectively [2]. Three loci, WDR36, MYOC and OPTN, have been identified as main causative genes, contributing less than $10 \%$ of all POAG etiology with WDR36 accounting for $5 \%$ alone $[2,5]$. WDR36 gene was identified within GLC1G locus on 5q22.1, associated with optic neuropathies in glaucoma. Although detailed pathogenesis of mutation of WDR36 remains unclear, genetic screening of WDR36 gene is believed to be useful for early diagnosis of presymptomatic open-angle glaucoma in high risk individuals [9].

In this study, we focus on WDR36 gene, investigating its role in JOAG, although other 6 genes have been reported to be associated with glaucoma, including myocilin (MYOC), optineurin (OPTN), neurotrophin 4 (NTF4), optic atrophy 1 (OPA1), cytochrome P450, family 1, subfamily B (CYP1B1), and latent transforming growth factor-beta binding protein 2 (LTBP2) [10].

The Next-Generation Sequencing (NGS), also named Massive Parallel Sequencing, is a high-throughput DNA sequencing technology that enables scientists to analyze whole genomes or transcriptomes in a more precise but rapid manner, making great advance in genetics or in other related fields [11]. It is believed that more detailed genomic alteration could be detected precisely by NGS than by other conventional technologies, improving and renewing our understanding of genomic biology [12].

To the best of our knowledge, the causative genes OPTN and MYOC, bar WDR36, have been studied already in Taiwan [3, 13]. In this study, we aimed to compare variants in WDR36 genes of JOAG patients with those of unrelated normal individuals in Taiwan, using NGS technology and comparative genetic analysis methods. Understanding the genetic correlation between JOAG and WDR36 may have benefits to early diagnosis or treatment of this disease.

\section{Materials and Methods}

\section{Subject Selection}

This part of subject selection is partially modified out of a previous study investigating the same set of 1210 patients for juvenile-onset open-angle glaucoma research [13]. In addition to the original subjects, 10 new patients who had undergone identical clinical examinations, including IOP measurement, visual-field test, slit-lamp examination and fundus examination, were added to this study.

Identification of JOAG can be established as clinical presentations of intraocular pressure (IOP) $>$ $22 \mathrm{mmHg}$, a cup-to-disc ratio $>0.5$ or one with an asymmetric appearance, a visual field loss characteristic of glaucomatous change, and an open angle width ranging from Shaffer grade II to IV without any other apparent secondary cause like trauma or surgery. In total, 61 unrelated individuals screened were diagnosed as JOAG.

Randomly selected, another 61 individuals over 50 years old comprised the control group. Subjects of the control group also received aforementioned examinations, and glaucoma-free outcomes of the subjects were confirmed. All of 122 individuals, including 61 patients and 61 controls, belong to Han ethic origin in the study. The study protocol was approved by the Institutional Review Board of the Kuo General Hospital, conforming to the World Medical Association's Declaration of Helsinki (2000). All of the participants provided signed informed consent to participate in the study after the details of the study had been explained to them.

\section{Detection of the mutations of the WDR36 gene}

Genomic DNA samples were extracted from $10 \mathrm{ml}$ peripheral blood collected from every participating individual and were purified by using a Gentra DNA Blood Kit (Gentra Systems, Inc. Minneapolis, USA) according to the manufacturer's instructions. Gel electrophoresis and spectrophotometry were applied to determine the quality and quantity of the purified DNA, respectively. Mutations in the 23 exons and flanking introns of the WDR36 gene were screened by SOLiD ${ }^{\mathrm{TM}}$ NGS sequencing (Ensembl Transcript ID. ENST00000323652).

Long range polymerase chain reaction (PCR) was applied for enrichment of WDR36 gene, intragenic primer sequences shown in Table 1. Cycling profile of long range PCR was conducted as follows: one cycle at $94^{\circ} \mathrm{C}$ for $2 \mathrm{~min}$, followed by 10 cycles at $94^{\circ} \mathrm{C}$ for $10 \mathrm{~s}$, at $55^{\circ} \mathrm{C}$ for $30 \mathrm{~s}$, and at $68^{\circ} \mathrm{C}$ for $14 \mathrm{~min}$, followed by 30 cycles at $94^{\circ} \mathrm{C}$ for $10 \mathrm{~s}$, at $55^{\circ} \mathrm{C}$ for $30 \mathrm{~s}$, and at $68^{\circ} \mathrm{C}$ for $14 \mathrm{~min}(+20 \mathrm{~s}$ every cycle), followed by a final extension step at $72^{\circ} \mathrm{C}$ for $5 \mathrm{~min}$, according to SequalPrep ${ }^{\mathrm{TM}}$ Long PCR Kit protocol (Invitrogen, USA). According to the manufacturer's instructions, PCR products were cleaned up using AMPure Reagent beads (Agencourt, Beverly, MA). Amplicons were pooled at an equimolar ratio, and a sequencing library was made up of 5 micrograms of DNA.

Generally, SOLiD Sequencing Analysis was then performed with the amplified genomic DNA, with the first step being preparation of fragment library, followed orderly by preparation of templated bead, SOLiD analyzer operation (sequencing) and data analysis. 
Table 1. Oligonucleotide Primer Pairs for PCR

\begin{tabular}{ll}
\hline Name & Oligonucleotide \\
\hline WDR36 E1F & GGGTCTGTGAGTGAATCCCTCTGTC \\
WDR36 E5R & CCACAACTGCAGGCTTCCTTG \\
WDR36 E5F & CAAGGAAGCCTGCAGTTGTGG \\
WDR36 I10R & GCATTGATGAAACTTCCTCCAGTTG \\
WDR36 I10F & CAACTGGAGGAAGTTTCATCAATGC \\
WDR36 I15R & TGATCGCATCAACTCCCTGAAA \\
WDR36 I15F & TTTCAGGGAGTTGATGCGATCA \\
WDR36 I16R & CAACAGGGAGGAAACAGGAGGA \\
WDR36 I16F & TCCTCCTGTTTCCTCCCTGTTG \\
WDR36 I19R & AGCACCCTTGCCGATAAGGC \\
WDR36 I19F & GCCTTATCGGCAAGGGTGCT \\
WDR36 E23R & CTCCCAGAATGTCAAAAAGTG \\
\hline
\end{tabular}

\section{Splicing site prediction by neural network}

Both normal and variant WDR36 genomic sequence of the intron 3 were analyzed by using a neural network prediction system of Berkeley Drosophila Genome Project to predict whether there were novel donor or acceptor sites [14].

\section{Result}

We screened the WDR36 (GenBank NC_000005) of 61 JOAG patients and 61 normal Taiwanese individuals, including intronic sequence, promoter region and coding region, by PCR amplification and targeting genome sequencing analysis. In general, 27 variants were identified from 61 JOAG patients, whereas 6 variants were identified from the control group. Only one variant c.1964+2910T $>$ C was found in both JOAG patients and normal controls, but no significant difference in frequency was shown between the two groups, with the $\mathrm{p}$ value calculated by $\chi^{2}$ test being 0.862 , suggesting that this variant has no direct relation to JOAG pathogenesis (Table 2).

Table 2. c. 1964+2910T>C Variant Shared by JOAG Patients and Normal Individuals

\begin{tabular}{llll}
\hline & $\begin{array}{l}\text { Reference Allele } \\
\text { Count }\end{array}$ & Novel Allele Count & $\begin{array}{l}\text { Novel Allele } \\
\text { Frequency }\end{array}$ \\
\hline JOAG & 2 & 3 & 0.600 \\
Control & 27 & 37 & 0.578 \\
p value & 0.862 & & \\
\hline
\end{tabular}

Among the 26 variants only found in the JOAG patients, 20 of them were established SNPs, including rs1971050, rs1993465, rs13153937, rs199945131, rs201148106, rs6859041, rs1379298, rs10038177, rs6865932, rs10045255, rs10043631, rs10038058, rs13178997, rs4957924, rs43203, rs6594498, rs7722241, rs7702774, rs4530809 and rs12520738 (Table 3). Among those 20 SNPs, we found that only the rs13178997 (c.1494+1111G>T) had a significant difference in frequency between JOAG patients and reference from NCBI database $(p<0.02)$, while there was another SNP, rs10038177 (c.710+30C >T), which had been reported to be a risk factor for high-tension glaucoma [15]. The remaining $18 \mathrm{SNPs}$ had no remarkable findings by comparing to the corresponding frequencies on NCBI database.

Table 3. Variants Found Only in Normal Individuals

\begin{tabular}{llllll}
\hline HGVS Name & Allele Count & Coordinate & $\begin{array}{l}\text { Chromosome } \\
\text { Position } \\
\text { (GRCh38.p2) }\end{array}$ \\
\hline c.1262-223 G>A & G:0.71 1589 & 13664 & 111105834 & rs2034896 \\
& A:0.29 647 & & & \\
c.1964+2843 A>G & A:0.34 11 & 23825 & 111115996 & - \\
& G:0.66 & 21 & & & \\
c.1964+2865 G>C & G:0.71 15 & 23847 & 111116018 & - \\
& C:0.29 & 6 & & & \\
c.1965-2867 A>G & A:0.80 & 156 & 23875 & 111116146 & - \\
& G:0.20 & 40 & & & \\
c.1964+2918 C>T & C:0.16 9 & 23900 & 111116071 & - \\
& T:0.84 46 & & & \\
\hline
\end{tabular}

As for the other 6 novel variants, including c. $460-650 A>G, \quad$ c. $1495-807 \mathrm{~T}>\mathrm{G}, \quad$ c. $1965-2849 \mathrm{~A}>\mathrm{G}$, c. $1965-2833 C>$ T, c. $1965-2812 A>G$ and c. 1965-2786C $>\mathrm{G}$ were only found in the JOAG patients, none of them were established SNPs (Table 4). In order to study the influence of the 6 novel variants on mRNA transcription, we utilized a neural network prediction system to predict whether there is any splicing alteration, using a cutoff of minimal score by 0.4 . The variant c.460-650A $>\mathrm{G}$ was the only one of the six that creates a novel acceptor site AG at c.460-644_643AG, located within intron 3 (Figure 1). The neural network value of the novel acceptor splicing site score was 0.5, crossing the threshold cutoff. The coding DNA sequence (CDS) of WDR36 was translated into 952 amino acids under normal condition, whereas in c. $460-650 A>G$ variants, the mRNA were added with 642 nucleotides within intron 3, leading to a premature termination of translation at residue 198 (TGA) (Figure 1). Therefore, we suggest that this variant c.460-650A $>\mathrm{G}$ can cause the production of truncated proteins and further impair the function of the proteins by altering the splicing pattern, which might be one of the etiologies of JOAG.

Another 2 SNPs found in JOAG patients, c.710+30C $>\mathrm{T}$ (rs10038177), reported to be a risk factor of high-tension glaucoma, and c.1494+1111G $>\mathrm{T}$ (rs13178997), calculated to have significant difference in frequency between JOAG patients and the control group, were also analyzed by the neural network prediction system in the same way, which revealed no difference of splicing result in c.710+30C $>\mathrm{T}$ (rs10038177). A minimal alteration of increased score of an acceptor site from 0.81 to 0.91 was detected in c.1494+1111G>T (rs13178997) (Table 5). 


\section{Discussion}

The WDR36 gene was recognized as one of the major causative genes of POAG. Similarly, in a yeast model, WDR36 sequence variant made a functional defect in rRNA processing that may have contributed to the development of POAG [16]. However, the role of WDR36 in POAG is open to debate. Gallenberger $e t$ al. described in 2014 that, despite the decrease in the expression of Wdr36 mRNA, heterozygote Wdr36-deficient mice showed no significant difference from wild type individuals with regards to structures, intraocular pressure, susceptibility of retinal ganglion cells to excitotoxic damage, susceptibility of optic nerve to high IOP damage, and analysis of rRNA processing [17]. Moreover, the role of WDR36 in POAG had been studied in different populations. Mookherjee and colleagues investigated 10 SNPs proposed to be associated with POAG in eastern India, and found that there was little correlation between POAG and SNPs in WDR36, except for rs10038177 (c.710+30C > T) which may be a risk factor for high tension glaucoma [15]. In Italian population, it was described that WDR36 sequence variances play a minor role in the pathogenesis of POAG [18]. Also, WDR36 was reported to be irrelevant to the development of glaucoma among German population [19].

Table 4. Variants Found in JOAG Patients

\begin{tabular}{|c|c|c|c|c|c|c|c|c|}
\hline \multirow{2}{*}{$\begin{array}{l}\text { HGVS Name } \\
\text { c. } 330+925 \mathrm{C}>\mathrm{T}\end{array}$} & \multirow{2}{*}{$\begin{array}{l}\text { Coordinate } \\
1372\end{array}$} & \multirow{2}{*}{$\begin{array}{l}\text { Position } \\
\text { intron1 }\end{array}$} & \multirow{2}{*}{$\begin{array}{l}\text { Chromosome Position } \\
\text { (GRCh38.p2) } \\
111093543\end{array}$} & \multirow{2}{*}{$\begin{array}{l}\text { SNP } \\
\text { rs1971050 }\end{array}$} & \multicolumn{2}{|c|}{ Allele Count and Frequency } & \multirow{2}{*}{$\begin{array}{l}\text { Reference Allele } \\
\text { Frequency } \\
0.389\end{array}$} & \multirow{2}{*}{$\begin{array}{l}\mathrm{p} \text { value } \\
0.208\end{array}$} \\
\hline & & & & & 11547 & C: 0.55 & & \\
\hline & & & & & 9448 & $\mathrm{~T}: 0.45$ & 0.611 & \\
\hline \multirow[t]{2}{*}{ c. $459+221 \mathrm{~A}>\mathrm{G}$} & 5229 & intron3 & 111097400 & rs1993465 & 8871 & A: 0.69 & 0.667 & 0.861 \\
\hline & & & & & 4023 & G: 0.31 & 0.333 & \\
\hline \multirow[t]{2}{*}{ c. $460-113 \mathrm{G}>\mathrm{A}$} & 6438 & intron3 & 111098609 & rs13153937 & 14732 & G: 0.75 & 0.686 & 0.578 \\
\hline & & & & & 4907 & A: 0.25 & 0.314 & \\
\hline \multirow[t]{2}{*}{ c. $577+624 \mathrm{G}>\mathrm{T}$} & 7292 & intron 4 & 111099463 & rs199945131 & 15 & G: 0.01 & - & - \\
\hline & & & & & 1112 & T: 0.99 & - & \\
\hline \multirow[t]{2}{*}{ c. $577+637 \mathrm{~T}>\mathrm{G}$} & 7305 & intron 4 & 111099476 & rs201148106 & 983 & T: 0.55 & - & - \\
\hline & & & & & 793 & G: 0.45 & - & \\
\hline \multirow[t]{2}{*}{ c. $577+694 \mathrm{G}>\mathrm{A}$} & 7362 & intron 4 & 111099533 & rs6859041 & 8768 & G: 0.72 & 0.667 & 0.670 \\
\hline & & & & & 3455 & A: 0.28 & 0.333 & \\
\hline \multirow[t]{2}{*}{ c. $578-561 \mathrm{~T}>\mathrm{C}$} & 7857 & intron 4 & 111100028 & rs1379298 & 11861 & T: 0.71 & 0.617 & 0.458 \\
\hline & & & & & 4918 & C: 0.29 & 0.383 & \\
\hline \multirow[t]{2}{*}{ c. $710+30 \mathrm{C}>\mathrm{T}$} & 8581 & intron5 & 111100751 & rs10038177 & 2932 & C: 0.77 & 0.637 & 0.268 \\
\hline & & & & & 892 & $\mathrm{~T}: 0.23$ & 0.363 & \\
\hline \multirow{2}{*}{ c. $710+432 G>C$} & 8983 & intron5 & 111101153 & rs6865932 & 12948 & G: 0.69 & 0.663 & 0.809 \\
\hline & & & & & 5781 & C: 0.31 & 0.338 & \\
\hline \multirow[t]{2}{*}{ c. $765+259 A>G$} & 10488 & intron6 & 111102658 & rs10045255 & 8709 & A: 0.70 & 0.663 & 0.765 \\
\hline & & & & & 3757 & G: 0.30 & 0.337 & \\
\hline \multirow{2}{*}{ c. $1494+90 \mathrm{C}>\mathrm{T}$} & 15359 & intron 12 & 111107529 & rs10043631 & 5403 & C: 0.76 & 0.617 & 0.221 \\
\hline & & & & & 1688 & T: 0.24 & 0.383 & \\
\hline \multirow[t]{2}{*}{ c. $1494+143 A>G$} & 15412 & intron 12 & 111107582 & rs10038058 & 4633 & A: 0.65 & 0.651 & 0.976 \\
\hline & & & & & 2524 & G: 0.35 & 0.349 & \\
\hline \multirow[t]{2}{*}{ c. $1494+1111 \mathrm{G}>\mathrm{T}$} & 16380 & intron 12 & 111108550 & rs13178997 & 6059 & G: 0.75 & 0.434 & 0.011 \\
\hline & & & & & 1995 & $\mathrm{~T}: 0.25$ & 0.566 & \\
\hline \multirow[t]{2}{*}{ c. $2316+256 \mathrm{C}>\mathrm{T}$} & 29226 & intron19 & 111121397 & rs4957924 & 4948 & C: 0.54 & 0.384 & 0.214 \\
\hline & & & & & 4172 & T: 0.46 & 0.616 & \\
\hline c. $2316+1144 \mathrm{G}>\mathrm{A}$ & 30114 & intron19 & 111122285 & rs43203 & 5569 & G: 0.61 & 0.622 & 0.901 \\
\hline & & & & & 3613 & A: 0.39 & 0.378 & \\
\hline c. $2317-1152 \mathrm{G}>\mathrm{A}$ & 30482 & intron19 & 111122653 & rs6594498 & 11061 & G: 0.78 & 0.617 & 0.158 \\
\hline & & & & & 3069 & A: 0.22 & 0.383 & \\
\hline c. $2437-559 \mathrm{~T}>\mathrm{A}$ & 32878 & intron 21 & 111125049 & rs7722241 & 7100 & T: 0.73 & 0.711 & 0.885 \\
\hline & & & & & 2657 & A: 0.27 & 0.289 & \\
\hline c. $2437-455 \mathrm{G}>\mathrm{T}$ & 32982 & intron 21 & 111125153 & rs7702774 & 4397 & G: 0.70 & 0.705 & 0.961 \\
\hline & & & & & 1891 & T: 0.30 & 0.295 & \\
\hline c. $2707-202 A>G$ & 34361 & intron 22 & 111126532 & rs4530809 & 3968 & A: 0.71 & 0.711 & 0.989 \\
\hline & & & & & 1626 & G: 0.29 & 0.289 & \\
\hline c. ${ }^{*} 1045 \mathrm{~T}>\mathrm{G}$ & 35757 & exon23 & 111127928 & rs12520738 & 585 & T: 0.64 & - & - \\
\hline & & & & & 323 & G: 0.36 & - & \\
\hline c. $460-650 A>G$ & 5901 & intron3 & 111098072 & - & 773 & A: 0.36 & - & - \\
\hline & & & & & 1391 & G: 0.64 & - & \\
\hline c. $1495-807 \mathrm{~T}>\mathrm{G}$ & 17211 & intron 12 & 111109382 & - & 815 & T: 0.64 & - & - \\
\hline & & & & & 463 & G: 0.36 & - & \\
\hline c. $1965-2849 A>G$ & 23993 & intron 16 & 111116164 & - & 2 & A: 0.10 & - & - \\
\hline & & & & & 18 & G: 0.90 & - & \\
\hline c. $1965-2833 \mathrm{C}>\mathrm{T}$ & 24009 & intron16 & 111116180 & - & 249 & C: 0.54 & - & - \\
\hline
\end{tabular}




\begin{tabular}{|c|c|c|c|c|c|c|c|c|}
\hline & & & & & 211 & T: 0.46 & - & \\
\hline \multirow[t]{2}{*}{ c. $1965-2812 A>G$} & 24030 & intron16 & 111116201 & - & 234 & A: 0.47 & - & - \\
\hline & & & & & 263 & G: 0.53 & - & \\
\hline \multirow[t]{2}{*}{ c. $1965-2786 C>G$} & 24056 & intron16 & 111116227 & - & 1 & C: 0.02 & - & - \\
\hline & & & & & 50 & G: 0.98 & - & \\
\hline \multirow[t]{2}{*}{ c. $1964+2910 \mathrm{~T}>\mathrm{C}$} & 23892 & intron16 & 111116063 & - & 2 & $\mathrm{~T}: 0.40$ & - & - \\
\hline & & & & & 3 & C: 0.60 & - & \\
\hline
\end{tabular}

Table 5. Splicing Site Prediction Results of WDR36 in Variants and Control Group

\begin{tabular}{|c|c|c|c|c|}
\hline Variant & Novel acceptor site prediction & Score & Start & End \\
\hline \multirow[t]{2}{*}{ c. $460-650 A>G$} & ataaaggacctttAtgcacAGgtgtggtcagggttagggga & 0.34 & 348 & 388 \\
\hline &  & 0.50 & & \\
\hline \multirow[t]{2}{*}{ c. $1494+1111 \mathrm{G}>\mathrm{T}$} & ttctatcttacgctcccGtAGggacatttacctacatagtt & 0.81 & 484 & 524 \\
\hline & ttctatcttacgctcccTtAGggacatttacctacatagtt & 0.91 & & \\
\hline
\end{tabular}

A

$\begin{array}{llr}\text { Sequence } & \text { Score } \\ \text { Normal } & \text { gacctttAtgcacAGgtgtggtcag } & 0.34 \\ \text { Variant } & \text { gaccttGtgcacAGgtgtggtcag } & 0.50 \\ & \bigcup_{\text {c. } 460-650 \mathrm{~A}>\mathrm{G}} & \end{array}$

B c.459 aaggacctttatgcacaggtgtggtcagggttaggggaacaaaata ggggtggggaggcgtcagacac...(542)...catgatgactgagtaataa tatgaaattcttttaaacttcgatgttttagatagtacatacctttaaggg c.460

C

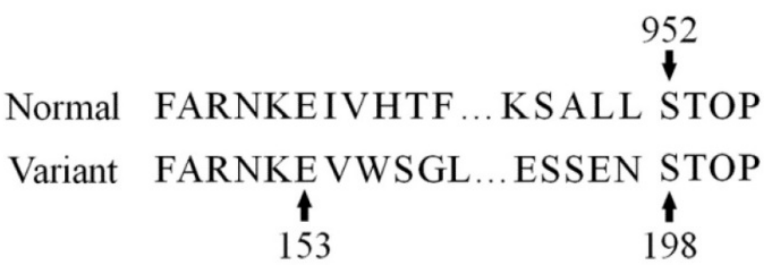

Figure 1. Spliced site prediction result of variation c. $460-650 A>G$ by a neural network prediction system. A: In WDR36, variant c.460-650A>G compared to normal transcript created a novel acceptor site c.460-644_643AG (star) with the score of neural network value increased from 0.34 to 0.50 . B: In variants c.460-650A $>$ G of WDR36, a 642-nucleotide fragment would be spliced out, leaving 38 residual nucleotides between c. 459 and c. 460 . To avoid redundancy, 542 nucleotides within the spliced fragment are abbreviated. C: Comparison of predicted protein sequence of normal and variant of WDR36 showed substantially shortened amino acid sequence with a premature termination.

Mookherjee and colleagues had studied 10 SNPs of WDR36 (rs1971050, rs1993465, rs13153937, rs10038177, rs11241095, rs10043631, rs10038058, rs10491424, rs17553936, and rs13186912) in East Indian population that were suspected to be contributive to POAG [15]. In comparison, 4 SNPs (rs13186912, rs17553936, rs10491424 and rs11241095) were not identified in our patients of POAG, suggesting that these 4 variants were merely genetic polymorphisms between Indian and Taiwanese populations, with minimal role in pathogenesis of POAG.

In different ethnic groups, the frequency of WDR36 variant varies greatly, ranging from $2.9 \%$ to $5.6 \%$ [10]. Moreover, different WDR36 mutations were identified among studies. For examples, Huang et al. identified several mutations within WDR36 which were mainly exons and were not found in our patients [10]. Likewise, Bao Jian Fan et al. did not find significant mutations in coding exons or splicing junctions of WDR36 associated with POAG [1]. We agree with the idea that, in consistency with Jonathan et al. [20], WDR36 may be just a modifier gene of POAG, playing a causative role in the pathogenesis of POAG to an uncertain extent.

WDR36, resembling yeast Utp21p in structure, with 14 WD40 repeats folded into two connected seven-bladed $\beta$-propellers, is known to be an essential nuclear protein localized in nucleolus that plays a critical role in the processing of $18 \mathrm{~S}$ rRNA [16, 21]. The variety and importance of function of WDR36 had been studied. Besides heart, brain and other non-ocular organs, expression of WDR36 were found in many of the ocular tissues, including lens, retina, optic nerve, and so on [9]. With WDR36 gene deleted or depletion by RNA interference, mouse embryos die before implantation and, in human trabecular meshwork cells, apoptosis is triggered and the formation of small subunit ribosomal RNA delays [21]. In zebrafish models, the function of Wdr36 protein had also been studied, and it was reported that Wdr36 interacted with the p53 pathway. In POAG, apoptosis causes the loss of retinal ganglion cells, which may be in accordance with the interaction between WDR36 and p53 pathway. However, without sufficient evidence, the WDR36 variant alone might not be a decisive factor in the pathogenesis of POAG, but its contributory role in the disease had be discussed [20]. Moreover, WDR36 was investigated by cell experiments. It had been described that WDR36 acted as a new scaffold protein, interacting with $\beta$ isoform of thromboxane A2 receptor, and enhancing the interaction between Gaq and PLC $\beta$. Despite of the unclear association between the findings and POAG, understanding the functions of WDR36 can benefit future studies to elucidate its role in POAG [22]. 
According to NCBI database, within the amino acid sequence translated from WDR36 mRNA, it has been established that there are 2 types of conserved domain, including Utp21 and WD40. Utp21, known as an rRNA-processing protein, is one of the components of U3 snoRNP which plays an important role in $18 \mathrm{~S}$ rRNA synthesis [16]. WD40, typically featuring 11-24 residues of $\mathrm{GH}$ dipeptide at its $\mathrm{N}$-terminus and 40 residues of $\mathrm{WD}$ dipeptide at its $\mathrm{C}$-terminus, is able to form a special propeller-like platform structure and can be found within many proteins of various functions, including signal transduction, pre-mRNA processing and cytoskeleton system [23]. The Utp21 conserved domain was found to be located at residue 728-948, whereas the residues associated with WD40 were identified at residue 155-420, 157-582, 332-650, 567-717 and 615-692, all of which were involved in regions behind 198, suggesting that, in c.460-650A>G variant, the premature termination at 198 residue could lead to the loss of these conserved domains, probably inducing the apoptosis of retinal ganglion cells which is one of the pathological features of POAG.

\section{Conclusions}

In summary, first, we have found a mutation c.460-650A $>\mathrm{G}$ within WDR36 which is likely to cause abnormal splicing and thus produce truncated proteins without conserved domains in Taiwanese population; further functional analysis of the mutations in this study is necessary in order to determine their exact role in POAG pathogenesis, which is also the limitation of this study. Second, by reviewing the literatures from different countries, we suggest that WDR36 is only a subordinate factor playing a role of modifier in POAG, without consistency among races or populations.

\section{Acknowledgements}

We would like to thank all of the subjects who participated in the present project. This work is supported by Chi-Mei Medical Center Liouying Research Grant (CLFHR 9929) and Chung Shan Medical University (CSMU-INT-102-04). Author contributions: Conceived and designed the experiments: JJY, SYL. Performed the experiments: HAS, YCY. Collected clinical samples: YCY. Analyzed the data: HAS, JJY. Wrote the manuscript: HAS, JJY.

\section{Competing Interests}

The authors have declared that no competing interest exists.

\section{References}

1. Fan BJ. Gene mapping for primary open angle glaucoma. Clinical biochemistry 2006; 39(3): 249 .

2. Weinreb RN, Khaw PT. Primary open-angle glaucoma. Lancet 2004; 363: 1711-1720.

3. Yen YC, Yang JJ, Chou MC, Li SY. Identification of mutations in the myocilin (MYOC) gene in Taiwanese patients with juvenile-onset open-angle glaucoma. Mol Vis 2007; 13: 1627-1634.

4. Drance S. Chronic open angle glaucoma: risk factors in addition to intraocular pressure. Acta Ophthalmol Scand 2001; 79: 545.

5. Fuse N. Genetic bases for glaucoma. Tohoku J Exp Med 2010; 221: 1-10.

6. Quigley HA, Broman AT. The number of people with glaucoma worldwide in 2010 and 2020. British journal of ophthalmology 2006; 90: 262-267.

7. Foster PJ, Oen FT, Machin D, Ng TP, Devereux JG, Johnson GJ, Khaw PT, Seach S.K. The prevalence of glaucoma in Chinese residents of Singapore: a cross-sectional population survey of the Tanjong Pagar district. Arch Ophthalmol 2000; 118: 1105-1111.

8. Tsai IL, Woung LC, Tsai CY, Kuo LL, Liu SW, Lin S, Wang IJ. Trends in blind and low vision registrations in Taipei City. Eur J Ophthalmol 2008; 18: 118-124.

9. Monemi S, Spaeth G, DaSilva A, Popinchalk S., Ilitchev E, Liebmann J, Ritch R, Heon E, Crick RP, Child A, Sarfarazi M. Identification of a novel adult-onset primary open-angle glaucoma (POAG) gene on 5q22.1. Hum Mol Genet 2005; 14725-733.

10. Huang X, Li M, Guo X, Li S, Xiao X, Jia X, Liu X, Zhang Q. Mutation Analysis of Seven Known Glaucoma-Associated Genes in Chinese Patients With GlaucomaMutations in Glaucoma Genes in Chinese Patients. Investigative ophthalmology \& visual science 2014; 55: 3594-3602.

11. Mardis ER. Next-generation DNA sequencing methods. Annu Rev Genomics Hum Genet 2008; 9: 387-402.

12. Friedman K, Resnick MB, Safran. Mutation Profiling of Clinically Advanced Cancers Using Next-Generation Sequencing for Targeted Therapy: A Lifespan Experience. R I Med J (2013), 2015; 98: 16-20.

13. Yen YC, Yang JJ, Chou MC, Li SY. Absence of optineurin (OPTN) gene mutations in Taiwanese patients with juvenile-onset open-angle glaucoma. Mol Vis 2008; 14: 487-494.

14. Reese MG, Eeckman FH, Kulp D, Haussler D. Improved splice site detection in Genie. Journal of computational biology 1997; 4: 311-323.

15. Mookherjee S, Chakraborty S, Vishal M, Banerjee D, Sen A, Ray K. WDR36 variants in East Indian primary open-angle glaucoma patients. Molecular vision 2011; 17: 2618

16. Footz TK, Johnson JL, Dubois S, Boivin N, Raymond V, Walter MA. Glaucoma-associated WDR36 variants encode functional defects in a yeast model system. Hum Mol Genet 2009; 18: 1276-1287.

17. Gallenberger M, Kroeber M, Marz L, Koch M, Fuchshofer R, Braunger BM, Iwata T, Tamm ER. Heterozygote Wdr36-deficient mice do not develop glaucoma. Exp Eye Res 2014; 128: 83-91.

18. Frezzotti P, Pescucci C, Papa FT, Iester M, Mittica V, Motolese I, Peruzzi S, Artuso R, Longo I, Mencarelli MA. Association between primary open-angle glaucoma (POAG) and WDR36 sequence variance in Italian families affected by POAG. British Journal of Ophthalmology 2010: bjo. 2009.167494.

19. Pasutto F, Mardin CY, Michels-Rautenstrauss K, Weber BH, Sticht H, Chavarria-Soley GRautenstrauss B, Kruse F, Reis A. Profiling of WDR36 missense variants in German patients with glaucoma. Investigative ophthalmology \& visual science 2008; 49: 270.

20. Skarie JM, Link BA. The Primary open-angle glaucoma gene WDR36 functions in ribosomal RNA processing and interacts with the p53 stress-response pathway. Human molecular genetics 2008; 17:2474-2485.

21. Gallenberger M, Meinel DM, Kroeber M, Wegner M, Milkereit P, Bosl MR, Tamm ER. Lack of WDR36 leads to preimplantation embryonic lethality in mice and delays the formation of small subunit ribosomal RNA in human cells in vitro. Hum Mol Genet 2011; 20: 422-435.

22. Cartier A, Parent A, Labrecque P, Laroche G, Parent JL. WDR36 acts as a scaffold protein tethering a G-protein-coupled receptor, Gaq and phospholipase $C \beta$ in a signalling complex. Journal of cell science 2011; 124: 3292-3304.

23. Smith TF, Gaitatzes C, Saxena K, Neer EJ. The WD repeat: a common architecture for diverse functions. Trends in biochemical sciences 1999; 24: 181-185. 